\title{
Pontos de Equilíbrio Hopf Subcríticos na Fronteira da Região de Estabilidade
}

\author{
Josaphat R. R. Gouveia Jr. ${ }^{1}$
}

Colegiado de Matemática, IFBA, Eunápolis, BA

Fabíolo Moraes Amaral ${ }^{2}$

Colegiado de Matemática, IFBA, Eunápolis, BA

Luís Fernando C. Alberto ${ }^{3}$

Departamento de Engenharia Elétrica e de Computação, EESC, USP, São Carlos, SP

Resumo. Uma caracterização completa da fronteira da região de estabilidade de uma classe de sistemas dinâmicos autônomos não lineares é desenvolvida admitindo a existência de pontos de equilíbrio não-hiperbólicos do tipo Hopf Subcríticos na fronteira da região de estabilidade. Sob a condição de transversalidade, mostra-se que a fronteira da região de estabilidade é composta pelas variedades estáveis de todos os pontos de equilíbrio presentes na fronteira da região de estabilidade, incluindo as variedades estáveis dos pontos de equilíbrio Hopf Subcríticos do tipo $k$, com $0 \leq k \leq n-2$, que pertencem à fronteira da região de estabilidade.

Palavras-chave. Sistemas Dinâmicos, Sistemas Não Lineares, Região de Estabilidade, Fronteira da Região de Estabilidade, Ponto de Equilíbrio Hopf Subcrítico.

\section{Introdução}

A modelagem de fenômenos dinâmicos, sejam eles sociais, econômicos, biológicos ou de engenharia, é realizada por conjuntos de equações diferenciais que usualmente dependem de um ou mais parâmetros. Ao variarmos um ou mais parâmetros de um sistema de equações diferenciais, mudanças topológicas podem ocorrer no comportamento das soluções desse sistema. Por exemplo, o ponto de equilíbrio de um sistema poderá perder estabilidade em determinados valores dos parâmetros do sistema e até mesmo, ao perder estabilidade, ocasionar o surgimento de órbitas periódicas isoladas, ou ciclos limites estáveis. Especificamente, a Teoria das Bifurcações permite-nos estudar como o sistema se comportará e em que momento perderá estabilidade ao variar um ou mais parâmetros do mesmo.

\footnotetext{
${ }_{1}$ josaphat@ifba.edu.br

${ }^{2}$ fabiolo@ifba.edu.br

${ }^{3}$ lfcalberto@usp.br
} 
Em sistemas elétricos de potência, por exemplo, o aparecimento de bifurcações sela-nó na fronteira da região de estabilidade como consequência da variação de carga foi relatado em [3]. Na análise da estabilidade de tensão em sistemas de energia, pontos de bifurcação de Hopf sobre a fronteira da região de estabilidade também foram encontrados, ver [6]. Em [4] foi apresentado um estudo do comportamento da região de estabilidade de sistemas dinâmicos autônomos não lineares e de sua fronteira, sujeitos a variações de parâmetros, quando da existência de uma bifurcação sela-nó do tipo zero na fronteira da região de estabilidade.

Neste trabalho são fornecidas condições necessárias e suficientes para que um ponto de equilíbrio Hopf subcrítico pertença à fronteira da região de estabilidade. Também é apresentada uma caracterização da fronteira da região de estabilidade de um ponto de equilíbrio assintoticamente estável na presença de pontos de equilíbrio Hopf subcríticos na fronteira da região de estabilidade.

\section{Preliminares}

Nesta seção, recordaremos alguns conceitos clássicos relacionados com a teoria de sistemas dinâmicos que são essenciais para o desenvolvimento posterior deste trabalho. Mais detalhes sobre os conteúdos explorados nesta seção podem ser encontradas em [10,14].

Consideremos o sistema dinâmico autonômo não linear:

$$
\dot{x}=f(x)
$$

onde $x \in \mathbb{R}^{n}$ e $f: \mathbb{R}^{n} \rightarrow \mathbb{R}^{n}$ é um campo vetorial suave. A solução de (1) iniciando em $x$ no tempo $t=0$ é denotada por $\varphi(t, x)$. Um conjunto $S \subset \mathbb{R}^{n}$ é dito ser um conjunto invariante de (1) se cada trajetória de (1) começando em $S$ permanece em $S$ para todo $t$.

O ponto $x^{\star} \in \mathbb{R}^{n}$ é um ponto de equilíbrio de (1) se $f\left(x^{\star}\right)=0$. Um ponto de equilíbrio $x^{\star}$ de (1) é dito ser hiperbólico se todos os autovalores da matriz Jacobiana $D_{x} f\left(x^{\star}\right)$ não têm parte real nula.

Além disso, um ponto de equilíbrio hiperbólico $x^{\star}$ é do tipo- $k$ se a matriz Jacobiana possui $k$ autovalores com parte real positiva e $n-k$ autovalores com parte real negativa.

Seja $x^{\star}$ um ponto de equilíbrio não-hiperbólico do sistema dinâmico autonômo não linear (1). Existem variedades invariantes suaves locais $W^{s}\left(x^{\star}\right), W^{c s}\left(x^{\star}\right), W^{c}\left(x^{\star}\right), W^{u}\left(x^{\star}\right)$ e $W^{c u}\left(x^{\star}\right)$ em $x^{\star}$, veja $[9,13]$. Estas variedades são chamadas de variedades estável, centro estável, central, instável e centro instável, respectivamente. As variedades estável e instável são únicas, mas as variedades centro estável, central e centro instável podem não ser.

Transversalidade é um conceito geométrico que trata das interseções das variedades e tem desempenhado um papel muito importante no desenvolvimento da teoria de sistemas dinâmicos. As variedades $M$ e $N$ de classe $C^{r}$, com $r \geq 1$, em $\mathbb{R}^{n}$, satisfazem a condição de transversalidade se ou (i) os espaços tangente de $M$ e $N$ geram o espaço tangente $\mathbb{R}^{n}$ em cada ponto $x \in M \cap N$, isto é, $T_{x}(M)+T_{x}(N)=T_{x}\left(\mathbb{R}^{n}\right)$ para todo $x \in M \cap N$ ou (ii) as variedades não se intersectam. 


\section{Ponto de Equilíbrio Hopf}

Nesta seção, um determinado tipo de ponto de equilíbrio não-hiperbólico, ou seja, o ponto de equilíbrio Hopf, é estudado. Em particular, o comportamento dinâmico em uma vizinhança deste equilíbrio é explorada em detalhes e o comportamento assintótico das soluções nas variedades locais invariantes é discutido.

Considere o sistema dinâmico não linear (1) e seja $p$ um ponto de equilíbrio não hiperbólico. Um ponto de equilíbrio não hiperbólico $p \in \mathbb{R}^{n}$ de (1) é chamado um ponto de equilíbrio de Hopf se as seguintes condições forem satisfeitas:

(i) $D_{x} f(p)$ tem um par simples de autovalores imaginários puros, $\pm i \omega$, e nenhum outro autovalor com parte real nula;

(ii) $l_{1} \neq 0$, onde $l_{1}$ é o Primeiro Coeficiente de Lyapunov, ver $[7,8]$.

Coeficientes de Lyapunov indicam o nível de degeneração do campo vetorial. Se o primeiro coeficiente de Lyapunov é diferente de zero, então o campo vetorial possui uma degeneração de ordem cúbica mostrando que termos cúbicos são os que determinam o tipo de comportamento dinâmico local na vizinhança do ponto de equilíbrio não hiperbólico na variedade central, ver [10] para mais detalhes.

Pontos de equilíbrio de Hopf podem ser classificados de acordo com o sinal do primeiro coeficiente de Lyapunov. Um ponto de equilíbrio de Hopf $p \in \mathbb{R}^{n}$ de (1) é chamado um ponto de equilíbrio Hopf supercrítico se o primeiro coeficiente de Lyapunov $l_{1}<0$ e é chamado um ponto de equilíbrio de Hopf subcrítico se o primeiro coeficiente de Lyapunov $l_{1}>0$.

Pontos de equilíbrio de Hopf podem também ser classificados em tipos de acordo com o número de autovalores de $D_{x} f(p)$ com parte real positiva. Um ponto de equilíbrio de Hopf $p$ de (1) é chamado um ponto de equilíbrio de Hopf do tipo- $k$ se $D_{x} f(p)$ tem $k(k \leq n-2)$ autovalores com parte real positiva e $n-k-2$ com parte real negativa.

Neste artigo, estamos principalmente interessados nos pontos de equilíbrio de Hopf subcríticos. Se $p$ é um ponto de equilíbrio de Hopf subcrítico do tipo- $k$, então as seguintes propriedades são satisfeitas, veja $[10,15]$ :

(i) A variedade instável $k$-dimensional $W^{u}(p)$ de $p$ existe, é única, e se $q \in W^{u}(p)$ então $\varphi(t, q) \longrightarrow p$ quando $t \longrightarrow-\infty$.

(ii) A variedade estável $(n-k-2)$-dimensional $W^{s}(p)$ de $p$ existe, é única, e se $q \in W^{s}(p)$ então $\varphi(t, q) \longrightarrow p$ quando $t \longrightarrow+\infty$.

(iii) A variedade centro instável $(k+2)$-dimensional $W^{c u}(p)$ de $p$ existe, é única, e se $q \in W^{c u}(p)$ então $\varphi(t, q) \longrightarrow p$ quando $t \longrightarrow-\infty$.

A Figura 1.1(a) ilustra as variedades invariantes para um ponto de equilíbrio de Hopf subcrítico do tipo-1 no $\mathbb{R}^{3}$ e a Figura 1.1(b) ilustra estas variedades invariantes para um ponto de equilíbrio de Hopf subcrítico do tipo-0 no $\mathbb{R}^{3}$.

\section{Caracterização da Fronteira da Região de Estabilidade}

Nesta seção, apresentaremos uma visão geral da teoria existente sobre a caracterização da fronteira da região de estabilidade de sistemas dinâmicos não-lineares. 


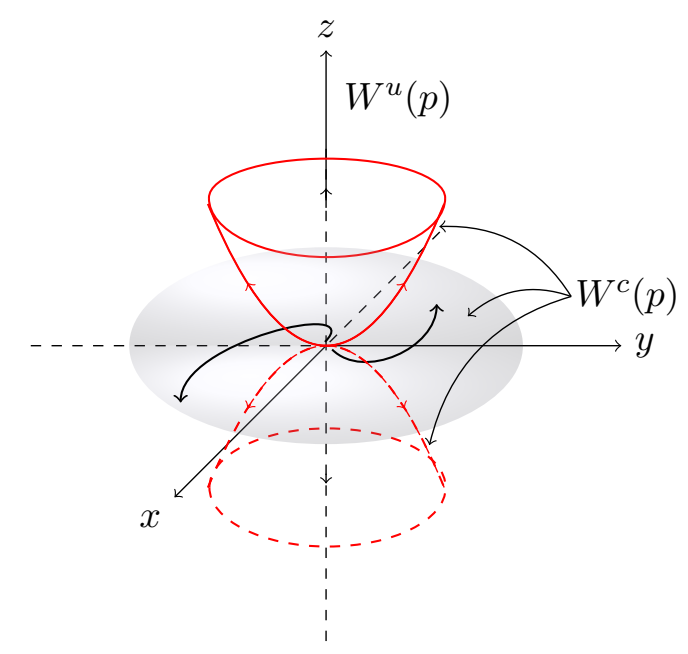

(a) Ponto de equilíbrio Hopf Subcrítico do tipo-1

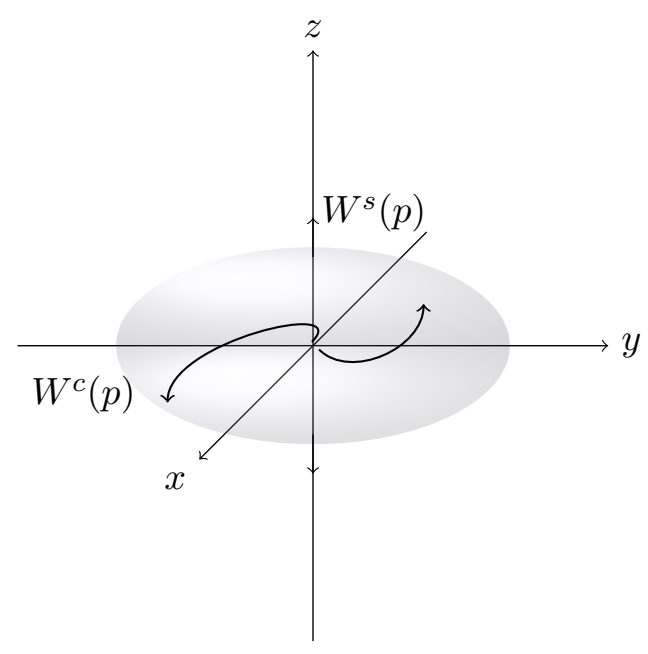

(b) Ponto de equilíbrio Hopf Subcrítico do tipo-

Figura 1: 1(a) Variedades $W^{c}(p)$ e $W^{u}(p)$ para um ponto de equilíbrio Hopf Subcrítico $p$ do tipo-1 do sistema (1) no $\mathbb{R}^{3}$. $W_{l o c}^{c}(p)$ não é única. Três possíveis escolhas para $W^{c}(p)$ são mostradas nesta figura. 1(b) Variedades $W^{c}(p)$ e $W^{s}(p)$ para um ponto de equilíbrio Hopf Subcrítico $p$ do tipo-0 do sistema (1) no $\mathbb{R}^{3}$. Neste caso, $W^{c}(p)$ é única.

Suponha que $x^{s}$ seja um ponto de equilívrio assintoticamente estável do sistema (1). A região de estabilidade (ou região de atração) de $x^{s}$ é o conjunto $A\left(x^{s}\right)=\left\{x \in \mathbb{R}^{n} \mid\right.$ $\varphi(t, x) \rightarrow x^{s}$ quando $\left.t \rightarrow+\infty\right\}$.

A região de estabilidade $A\left(x^{s}\right)$ é um conjunto aberto e invariante. Seu fecho $\overline{A\left(x^{s}\right)}$ é invariante e a fronteira da região de estabilidade $\partial A\left(x^{s}\right)$ é um conjunto fechado e invariante.

Com o intuito de compreender melhor a fronteira da região de estabilidade e obter melhores estimativas da região de estabilidade, foram desenvolvidos caracterizações da fronteira da região de estabilidade.

Uma caracterização completa da fronteira da região de estabilidade de um ponto de equilíbrio assintoticamente estável $x^{s}$ do sistema (1) foi desenvolvida em [2] sob as seguintes hipóteses:

(A1) Todos os pontos de equilíbrio em $\partial A\left(x^{s}\right)$ são hiperbólicos;

(A2) As variedades estável e instável dos pontos de equilíbrio em $\partial A\left(x^{s}\right)$ satisfazem a condição de transversalidade;

(A3) Trajetórias em $\partial A\left(x^{s}\right)$ aproximam-se de um dos seus pontos de equilíbrio quando $t \rightarrow \infty$.

A fronteira da região de estabilidade de um ponto de equilíbrio assintoticamente estável $x^{s}$ do sistema (1), satisfazendo as hipóteses (A1), (A2) e (A3), é a união das variedades estáveis de todos pontos de equilíbrio na fronteira $\partial A\left(x^{s}\right)=\bigcup_{i} W^{s}\left(x_{i}\right)$, onde $x_{i}, i=1,2, \ldots$ são os pontos de equilíbrio hiperbólicos na fronteira da região de estabilidade $\partial A\left(x^{s}\right)$.

As hipóteses (A1) e (A2) são propriedades genéricas de sistemas dinâmicos na forma de (1), veja [12]. Condições suficientes para a verificação da hipótese (A3) foram dadas 
em [1].

Neste artigo, estudaremos a caracterização da fronteira da região de estabilidade quando a suposição (A1) é violada. Em particular, estudaremos a caracterização da fronteira da região de estabilidade quando um ponto de equilíbrio não hiperbólico Hopf Subcrítico é encontrado na fronteira da região de estabilidade. Embora a suposição (A1) seja genérica, o estudo da caracterização da fronteira da região de estabilidade na presença de pontos de equilíbrio não-hiperbólicos é importante para entender o comportamento da região de estabilidade, como consequência de variações de parâmetros. Essas mudanças já foram investigadas na ocorrência de bifurcações sela-nó do tipo-zero na fronteira da região de estabilidade [4], [5], e na ocorrência de pontos de equilíbrio Hopf supercríticos do tipo $k, \operatorname{com} 1 \leq k \leq n-2,[7]$.

\section{Pontos de Equilíbrio de Hopf Subcrítico na fronteira da região de estabilidade}

Nesta seção, resultados inéditos de caracterização de equilíbrios na fronteira da região de estabilidade são apresentados. A caracterização da fronteira da região de estabilidade na presença de um ponto de equilíbrio Hopf Subcrítico será desenvolvida em dois passos. Primeiramente, estudaremos uma caracterização local da fronteira da região de estabilidade, estudando e caracterizando os pontos de equilíbrio que pertencem à fronteira da região de estabilidade, e em seguida, uma caracterização global será desenvolvida.

Os próximos teoremas oferecem condições necessárias e suficientes para garantir que um ponto de equilíbrio Hopf Subcrítico pertença à fronteira da região de estabilidade em termos das propriedades das suas variedades estável local, centro instável local e centro local.

Teorema 5.1. (Ponto de equilíbrio Hopf subcrítico na fronteira da região de estabilidade) Sejam $x^{s}$ um ponto de equilíbrio assintoticamente estável e $A\left(x^{s}\right)$ sua região de estabilidade. Seja $p$ um ponto de equilíbrio Hopf subcrítico de (1). Se p é um ponto de equilíbrio Hopf subcrítico tipo- $k$ de (1), com $0 \leq k \leq n-2$, então:

(i) $p \in \partial A\left(x^{s}\right) \Longleftrightarrow\left(W_{l o c}^{c u}(p) \backslash\{p\}\right) \cap \overline{A\left(x^{s}\right)} \neq \emptyset$

(ii) $p \in \partial A\left(x^{s}\right) \Longleftrightarrow W_{l o c}^{s}(p) \cap \partial A\left(x^{s}\right) \neq \emptyset$

A prova completa do Teorema 5.1 é longa e será omitida. A prova deste teorema é uma consequência do $\lambda$-lema para pontos de equilíbrio não hiperbólicos, veja [16]. Explorando esse lema, encontramos uma sequência de pontos na região de estabilidade convergindo para um ponto na variedade central do ponto de equilíbrio Hopf subcrítico.

Como uma consequência do Teorema 5.1, sabemos que $W_{l o c}^{c}(p) \cap A\left(x^{s}\right) \neq \emptyset$ se $k=0$ ou $W_{\text {loc }}^{c u}(p) \cap A\left(x^{s}\right) \neq \emptyset$ se $k \geq 1$ é uma condição suficiente para guarantir que um ponto de equilíbrio Hopf subcrítico $p$ pertença à fronteira da região de estabilidade. Será relevante estudar quando esta condição é também necessária.

O Teorema 5.1 pode ser melhorado impondo-se algumas condições para o campo vetorial. Sejam $x^{s}$ um ponto de equilíbrio assintoticamente estável, $p$ um ponto de equilíbrio Hopf Subcrítico de (1), e consideremos as seguintes suposições: 
(A1"') Todos os pontos de equilíbrio em $\partial A\left(x^{s}\right)$ são pontos de equilíbrio hiperbólicos ou pontos de equilíbrio do tipo Hopf subcrítico;

(A2") As variedades estável, instável, central e/ou centro instável dos pontos de equilíbrio em $\partial A\left(x^{s}\right)$ satisfazem a condição de transversalidade.

A condição (A1"') é mais fraca do que a condição (A1), uma vez que permite a presença de pontos de equilíbrio não hiperbólicos na fronteira da região de estabilidade.

O próximo resultado fornece condições necessárias e suficientes para garantir que os pontos de equilíbrio hiperbólicos e os pontos de equilíbrio Hopf Subcríticos pertençam à fronteira da região de estabilidade.

Teorema 5.2. (Pontos de equilíbrio tipo-k na fronteira de estabilidade): Seja $A\left(x^{s}\right)$ a região de estabilidade de um ponto de equilíbrio assintoticamente estável $x^{s}$ de (1) e suponha que as seguintes suposições (A1"'), (A2") e (A3) sejam satisfeitas. Sejam $p$ um ponto equilíbrio Hopf subcrítico do tipo- $k$, com $1 \leq k \leq n-3$, e $x^{\star}$ um ponto de equilíbrio hiperbólico do tipo- $k^{\prime}$, com $k^{\prime} \leq n$, de (1). Então:

(i) $p \in \partial A\left(x^{s}\right) \Longleftrightarrow W^{c u}(p) \cap A\left(x^{s}\right) \neq \emptyset$

$x^{\star} \in \partial A\left(x^{s}\right) \Longleftrightarrow W^{u}\left(x^{\star}\right) \cap A\left(x^{s}\right) \neq \emptyset$

(ii) $p \in \partial A\left(x^{s}\right) \Longleftrightarrow W^{s}(p) \subset \partial A\left(x^{s}\right)$

$x^{\star} \in \partial A\left(x^{s}\right) \Longleftrightarrow W^{s}\left(x^{\star}\right) \subset \partial A\left(x^{s}\right)$

O próximo teorema oferece uma caracterização completa da fronteira da região de estabilidade quando existem pontos de equilíbrio Hopf subcríticos em $\partial A\left(x^{s}\right)$.

Teorema 5.3. (Caracterização da Fronteira de Estabilidade): Seja $x^{s}$ um ponto de equilíbrio assintoticamente estável de (1) e $A\left(x^{s}\right)$ sua região de estabilidade. Se as suposições (A1"'), (A2") e (A3) são satisfeitas, então $\partial A\left(x^{s}\right)=\bigcup_{i} W^{s}\left(x_{i}\right) \bigcup_{j} W^{s}\left(p_{j}\right)$ onde $x_{i}$ são os pontos de equilíbrio hiperbólicos e $p_{j}$ os pontos de equilibrio Hopf subcríticos em $\partial A\left(x^{s}\right), i, j=1,2, \cdots$.

\section{Conclusão}

Neste artigo estudamos a caracterização da fronteira da região de estabilidade de sistemas dinâmicos não lineares autônomos na presença do ponto de equilíbrio Hopf Subcrítico. Foram oferecidas condições necessárias e suficientes para que um ponto de equilíbrio hiperbólico e um ponto de equilíbrio Hopf subcrítico pertença à fronteira da região de estabilidade. A caracterização da fronteira da região de estabilidade proposta neste trabalho é uma generalização das caracterizações encontradas na literatura permitindo a presença de um determinado tipo de ponto de equilíbrio não-hiperbólico na fronteira da região de estabilidade. Explorando as caracterizações desenvolvidas neste trabalho, esperamos no futuro próximo, entender como a região de estabilidade se comporta quando bifurcações locais do tipo Hopf ocorrem na fronteira da região de estabilidade. 


\section{Referências}

[1] H. D. Chiang, M. W. Hirsch and F. F. Wu, Stability region of nonlinear autonomous dynamical systems, IEEE Transactions on Automatic Control, vol. 33, 16-27, (1988).

[2] H. D. Chiang, F. F. Wu and P. P. Varaiya, Foundations of direct methods for power system transient stability analysis, IEEE Transactions on Circuits and Systems-I, vol. 34, 160-173, (1987).

[3] N. G. Bretas, A. C. P. Martins, L. F. C. Alberto and R. B. L. Guedes, Static simulation of voltage collapse considering the operational limits of the generators, Power Engineering Society General Meeting, IEEE, vol. 4, (2003).

[4] F. M. Amaral and L. F. C. Alberto, Stability boundary characterization of nonlinear autonomous dynamical systems in the presence of a type-zero saddle-node equilibrium point, TEMA, vol. 11, 111-120, (2010).

[5] F. M. Amaral and L. F. C. Alberto, Stability region bifurcations of nonlinear autonomous dynamical systems: Type-zero saddle-node bifurcations, IJRNC, vol. 21, 591-612, (2011).

[6] Y. Gao, Bifurcations and stability boundary of a power system, Acta Mathematicae Applicatae Sinica, Springer, vol. 20, n. 3, 513-532, (2004).

[7] J. R. R. Gouveia Jr., L. F. C. Alberto and F. M. Amaral, Supercritical Hopf equilibrium points on the Boundary of the Stability Region, CDC, (2013).

[8] J. R. R. Gouveia Jr., F. M. Amaral and L. F. C. Alberto, Stability boundary characterization of nonlinear autonomous dynamical systems in the presence of a supercritical Hopf equilibrium point, IJBC, vol. 23, (2013).

[9] M. W. Hirsch, C. C. Pugh and M. Shub, Invariant manifolds, Bull. Amer. Math. Soc., vol. 76, n. 5, 1015-1019, (1970).

[10] Y. A. Kuznetsov, Elements of applied bifurcation theory, Springer, (1995).

[11] J. Lee and H. D. Chiang, Stability regions of non-hyperbolic dynamical systems: theory and optimal estimation, IEEE International Symposium on Circuits and Systems, $28-31,(2000)$.

[12] J. Palis, On Morse-Smale dynamical systems, Topology, vol. 8, 385-405, (1969).

[13] L. Perko, Differential equations and dynamical systems, Springer, (1991).

[14] S. Smale, Differentiable dynamical systems, Bulletin of the American Mathematical Society, vol. 73, 747-817, (1967).

[15] J. Sotomayor, Generic bifurcations of dynamical systems, Dynamical Systems, New York: Academic Press, 549-560, (1973). 